\title{
LA FORMACIÓN DE PROFESORES ANTE LOS RETOS DE LA SOCIEDAD MULTICULTURAL
}

Luzia Alves de Carvalho*

\section{RESUMEN}

La sociedad multicultural es hoy un gran desafío a la educación intercultural. En efecto, el mundo se tornó pequeño: una plaza única donde se mueven gentes de todas las razas, culturas, etnias; un gran mercado donde transitan capital, tecnologías, recursos, empresas y productos. En estos contextos la educación tiene que cambiar y buscar modelos adecuados a la heterogeneidad vigente en toda escuela. Pero esta educación no se podrá llevar a cabo sin una sólida, actual y eficaz formación de los profesores, dotados de las competencias necesarias a la educación que respete las diferencias y garantice el aprendizaje.

\section{PALABRAS CLAVE}

Multiculturalismo. Interculturalismo. Reconstrucción social. Formación del profesorado

\begin{abstract}
The multicultural society is nowadays a great challenge to the intercultural education. Indeed. The world becomes small like an only square where people from all races, cultures and ethnic groups walk around. It is like a huge market where money, technologies, resources, enterprises and products circulate. In this context Education has to change and seek right models according to the current heterogeneity that exists in all the schools. However, this Education won't happen without a solid, up-to-date and efficient training of the teachers. These teachers should have a kind of competence which respect the differences and guarantee the learning demanded by the world in constant changing.
\end{abstract}

\section{KEY- WORDS}

Multiculturalism. Intercultural. Social reconstruction. Teacher's training.

* Doctoranda de Sociología de la UPSAM , y Coordinadora de Institutos Superiores de Educación de CENSA, Campos, Brasil. 


\section{INTRODUCCIÓN}

El mundo es una aldea global interrelacionada por los medios de comunicación, caracterizada por la integración, el universalismo y la globalización de la economía. (Calvo, 2000). Ésta viene provocando, a escala mundial, un triple movimiento de aceleración, de mundialización y de regionalización de los flujos de población.

El mundo se ha tornado pequeño, una plaza única, donde se mueven gentes de todas las razas y culturas; un gran mercado donde transitan capital, tecnología, recursos, empresas y productos. En este contexto, el proceso de aprendizaje de la convivencia entre personas de grupos culturales diferentes es función de la educación intercultural ${ }^{1}$, y se realiza mediante el desarrollo de elementos cognitivos y comunicativos facilitadores de las relaciones sociales en un plano de igualdad jurídica y práctica.

La educación monocultural no sólo no responde a las necesidades de socialización, sino que se convierte en obstáculo para el interculturalismo, puesto que éste precisa de relaciones sociales concretas y experiencias que materialicen los principios relacionales no excluyentes entre las personas de culturas y poblaciones humanas distintas.

El modelo monocultural ha de cambiarse, ya que no tiene sentido en el contexto de pluralidad cultural, y sus consecuencias afectan directamente a nuestro sistema educativo, llevando a replantearse las bases mismas de su trabajo de legitimación y transmisión cultural, tradicionalmente vinculadas a las pautas de la cultura mayoritaria. La escuela debe revisar su lógica de funcionamiento en el que tradicionalmente ha estado ausente el referente de los grupos y culturas minoritarios.

Ya afirmaba Lerena (1989, p.88): "A medida que la enseñanza deja de llevarse a cabo en un círculo familiar - esto es, en el círculo de los familiarizados con la particular cultura que la escuela está encargada de imponer e inculcar - el conflicto intercultural debe crecer".

Bajo el rótulo de "Educación Intercultural" encontramos una gama de modelos educativos según diferentes criterios: los objetivos que se plantean, el contexto en el que se aplican, los sujetos a que se dirigen, los principios ideológicos que los sostienen $-\mathrm{y}$ de acuerdo con los problemas específicos a los que pretenden dar repuestas.

Sabariego (2002: 79), Banks (1986, 1994), Etxeberria (1992), McCarthy (1994), Moñoz Sedano (1997), García Castaño et al (1997), Bartolomé (1997) son autores que presentan las tipologías más representativas en Educación Multicultural $^{2}$. Por su carácter sugerente e integrador de diferentes propuestas ya usadas por otros estudiosos enfocaremos la taxonomía de Bartolomé sobre programas de educación multicultural, (Sabariego, 2002: 83), como base para nuestra reflexión sobre formación de profesores para una educación intercultural. 
El primer enfoque, «hacia la formación hegemónica de la cultura del país de acogida», comprende los siguientes modelos: asimilacionista, compensatorio y segregacionista. El primer modelo incorpora el alumnado de minoría al sistema escolar normal, que no contempla las peculiaridades de la cultura de origen. El segundo, considera las diferencias como obstáculos al éxito escolar. El tercero relega a las minorías étnicas a escuelas especiales, impidiendo o dificultando su acceso al sistema educativo de la cultura dominante.

El segundo enfoque, "hacia el reconocimiento de la pluralidad de culturas", comprende el modelo de "currículo multicultural" que se modifica parcial o totalmente incluyendo contenidos multiculturales y el modelo de "pluralismo cultural" que reconoce el derecho a la expresión de las minorías étnicas en la escuela.

El tercer enfoque, "hacia una opción intercultural, basada en la simetría cultural", busca el desarrollo del autoconcepto, de los valores de la diferencia y diversidad, con los modelos de "orientación multicultural", "educación no-racista" e "intercultural".

El cuarto enfoque, "hacia una sociedad más justa, luchando contra la asimetría cultural, social y política" es un enfoque sociocrítico abarcando el modelo "holístico de Banks", el "antirracista" y el "radical".

El enfoque "global" es el que incluye la opción intercultural y la verdadera lucha contra toda discriminación. Intenta valorar la diversidad como la riqueza y promover la igualdad, educando para la ciudadanía.

Bartolomé intenta luchar contra la injusticia provocada por una asimetría cultural y la lucha contra toda discriminación. Sabemos, sin embargo, que los modelos basados en la hegemonía de la cultura, del reconocimiento de la pluralidad de culturas y la simetría cultural son todavía los más usados en las experiencias de educación hoy, en España. "En este contexto, la formación de los profesores es el "gran reto educativo que en los albores del siglo XXI, ha convertido la educación para la ciudadanía en una sociedad multicultural en un movimiento educativo igualmente relevante y complementario a la educación intercultural, para hacer realidad la convivencia en la diferencia y en un plano de igualdad y justicia social" (Banks en Sabariego, 2002: 84)

La educación intercultural incorpora la idea del compromiso como ciudadanos en la construcción de una sociedad humanamente global, con pleno sentido de sí, que se plantea desde un paradigma centrado en la alteridad, el diálogo y en los derechos humanos. Intenta la lucha contra todas las formas de exclusión social, el desarrollo de estrategias educativas que favorezcan la asunción de valores comunes compartidos y participación en un proyecto común donde cada persona tiene su lugar y su responsabilidad.

Ella asume los valores de la democracia moral, comprometidos con la promoción de la libertad, el respeto, la solidaridad, la justicia social y el desarrollo de nuevas pautas de convivencia social, valores arraigados en la ética básica 
(García Saez, 1998: 153) o ética mundial (Pérez de Cuellar: 1997). A partir de ahí se han planteado discursos y debates sobre el papel de la educación intercultural en la promoción de la interdependencia global y en el desarrollo de cambio de actitudes y comportamientos que reconozcan y promuevan la interdependencia y la cooperación.

En este sentido el alumnado debe aprender a imaginarse o prever de que manera el mundo puede convertirse en más pequeño y la humanidad en más grande, el modo de contribuir hacia la humanización de la sociedad, y la construcción de la ciudadanía mundial, a través de una educación para la paz, y el desarrollo interdependiente, una educación "internacional-global".

Se sabe que la llave de la verdadera educación intercultural es la formación del profesorado, (Sabariego, 2002). Pero, el problema es que, la teoría va muy por delante de la práctica y el profesorado no está recibiendo en su formación inicial una preparación en educación intercultural, ni tampoco la orientación suficiente en esta línea, dentro de las distintas modalidades de formación permanente (Jordan, 1995).

Según investigadores en este campo, la formación no está siguiendo algunos criterios uniformes que deberían ofrecer orientaciones conceptuales para la estructuración de los distintos programas.

La formación del profesorado que intentamos se centra en el paradigma crítico - reconstructivista, interviniendo en la concepción de currículo, del aprendizaje, de los métodos, de las actividades docentes y en los programas de formación.

\section{ENFOQUE DE RECONSTRUCCIÓN SOCIAL EN LA FORMACIÓN DEL PROFESORADO}

El enfoque de reconstrucción social en el paradigma de formación equivale a la orientación crítica, reconstructivista social de Zeichener, que destaca el compromiso de la enseñanza con la sociedad, poniendo énfasis en las habilidades del profesorado para valorar sus acciones, los contextos sociales, su contribución a la igualdad y a la justicia social.

La tradición reconstructivista social promueve en el profesorado una disposición contraria a la desigualdad. Incluye confrontar formas de opresión y dominación, como es el caso del sexismo, el racismo y el clasismo.

Gollinck afirma que pocos programas de formación del profesorado se apoyan en el reconstructivismo social. A su vez, García sostiene que el desarrollo de un currículo intercultural requiere la asunción de un compromiso ético y profesional por los profesores en su rol de despertar valores y actitudes dentro de una escuela plural y democrática, como agentes sociales de transformación. "Existe consenso entre los investigadores de la formación del profesorado que, para 
implementar la educación intercultural, se debe hacer cambios en el currículo, los recursos educativos, los estilos de enseñaza y aprendizaje, en las actitudes, percepciones, comportamientos del profesorado, en la administración, los objetivos, las normas y la cultura escolar" (Sabariego ,2002: 10).

Es necesario pues, ahondar en las características del modelo reconstructivista-social de Formación de Profesores. Junto a la integración de contenidos, utilizando ejemplos de varias culturas en el proceso educativo, urge también una Metodología que luche para la reducción de los prejuicios en clase, adopción de una pedagogía de la equidad y creación de una cultura escolar inclusiva, que garantice el éxito académico de todos.

No se puede olvidar, todavía, que la educación intercultural en el enfoque reconstructivista social se hace dentro y en un contexto sociopolítico, porque el antirracismo y el interculturalismo no gozarán de ninguna credibilidad mientras no formen parte de los valores y las reglas que ordenan las relaciones económicas, sociales, políticas y culturales.

Los profesores no pueden olvidar esa naturaleza política de la educación intercultural que coloca las cuestiones sociales en su centro. Deben saber que todo cambio educativo que no reconozca la desigualdad estructural y la estratificación social, por razón de género, clase, etnicidad y otras diferencias no es más que una ilusión.

La educación intercultural desafía y retrasa el racismo y todas las formas de discriminación en las escuelas y en la sociedad, acepta y afirma el pluralismo (étnico, racial, religioso, lingüístico y económico, entre otros) que se hace presente en la población escolar constituida por los padres, alumnos y sus comunidades. Penetra el currículo, las estrategias metodológicas, las interacciones alumno profesorado - padres y las concepciones acerca de la enseñanza-aprendizaje.

Sleeter hace una crítica a la educación intercultural derivada de las posturas conservadoras y radicales (Sabariego, 2002). Según él, los radicales ven la desigualdad como fenómeno estructural en el interior de las Instituciones Sociales, en beneficio de los intereses de la cultura mayoritaria, ofreciendo pocos beneficios al alumnado de minorías. Señala algunas conclusiones, retos y conceptualizaciones muy importantes para la formación de los profesores: configuración de una cultura pública, común, sensible no sólo a las minorías étnicas sino también a los colectivos económicamente pobres y a los socialmente discriminados por múltiplas razones. Educar para la ciudadanía, construyendo este "auténtico unun" en que todos pueden participar como ciudadanos, es el gran reto para la formación de los profesores. Considerar la perspectiva política concibiéndola más como una posibilidad de cambio en la jerarquía social y no tanto en el programa a ser implementado. Avanzar en el desarrollo de los análisis estructurales contextuales y focalizar la práctica educativa en esta dirección. Más que transmitir conocimientos, reflejar sobre las implicaciones sociales de éstos y criticarlos para transformar las estructuras jerárquicas de una sociedad desigual, empezando por la escuela. 
Se reivindica, pues, una formación del profesorado para el tratamiento de la diversidad cultural que vaya más allá de la transformación de contenidos, y que se encamine hacia la consecución de objetivos emancipadores vinculados al compromiso con los principios democráticos en una sociedad multicultural.

\section{EL ESTADO DE LA FORMACIÓN DEL PROFESORADO EN ESPAÑA}

Jordán (1998) señala que los esfuerzos para llevar a cabo una adecuada formación del profesorado en educación intercultural son esporádicos, fragmentados y escasos, por falta de legislación específica al respecto, y en consecuencia de un falta de compromiso político, por parte de la administración, sobre este ámbito. A falta de un marco legal e incentivos adicionales, los profesores que acuden a las ofertas de formación suelen ser en su mayoría voluntarios. Después de varias investigaciones sobre el estado de la situación, en cuestión de formación del profesorado, he obtenido algunos datos importantes:

1. Se hace necesaria una educación para la comprensión intercultural, principalmente en contexto de inmigración.

2. Se recomienda la inclusión de la dimensión intercultural y de la comprensión entre comunidades diversas en la formación inicial y continua.

3. Lo "legal" se extiende, en realidad, a algunas declaraciones de principios generales.

4. Las "iniciativas" de preparación en este campo, cuando se dan, suelen ser casi inevitablemente superficiales, puntuales, de sensibilización y optativas.

5. Los profesores que aceptan propuestas de formación suelen ser únicamente aquellos más sensibles a la problemática multicultural.

6. Falta acompañamiento en la práctica y ayuda especifica en las dificultades concretas del día a día.

7. Los profesores confiesan muy poca formación sistemática formal.

8. La repercusión de algunas jornadas de formación son casi nulas porque los talleres ofrecidos tienen poco significado para la realidad concreta del aula.

Las iniciativas de formación son bastante ineficaces porque son cortas, dirigidas sólo a los voluntarios, basadas casi exclusivamente en conferenciantes externos, con cuestiones que no interesan a los profesores, muchas veces apáticos, frustrados, indiferentes en cuanto a cursos y seminarios.

Según profesores participantes en iniciativas de formación intercultural, éstas no sólo resultan ineficaces sino que generalizan actitudes contrarias a la buena formación. Salen de ellas menos motivados, vacunados en relación a cualquier 
propuesta teórico-práctica. "La conclusión más clara y pesimista es que... la mayoría de los estudiantes de formación de profesorado no son capaces de abordar las complejidades asociadas con la enseñanza intercultural y que programas de formación del profesorado no son capaces de producir en los estudiantes el tipo de cambio de valores y disposiciones que son necesarios para una enseñanza eficaz con alumno de minorías étnicas y lingüísticas" (Marcelo en Jordán, 1998).

\section{ES NECESARIO CAMBIAR LA FORMACIÓN}

Según Bernabé (Nieto, 2002), sólo puede tener éxito si se basa en supuestos tales como: no dirigirse únicamente a profesores voluntarios o aislados, enfocar la formación en el staff escolar como "colectivo de educadores", abordar temas centrales de cada escuela concreta, reestructurar los procesos de funcionamiento, formar líderes con clara visión de lo que el profesorado intenta conseguir, impulsar la experiencia formativa, orientar para una visión de conjunto y para la cooperación. Incluye también, comprender la complejidad del hecho multicultural actual, descubrir sus propias contradicciones entre lo planteado en el proyecto educativo y la práctica habitual, una mayor sensibilidad para percibir la diversidad como una dimensión enriquecedora y no como déficit, llegar a aprender y a desarrollar prácticas y estrategias pedagógicas que favorezcan el proceso educativo intercultural, aprender a introducir a las familias del alumnado en la dinámica de la escuela, superando planteamientos asistenciales, favoreciendo una relación más participativa, para poder insertarse en la vida de la escuela e incluir el proyecto escolar en un proyecto social y político más amplio que envuelva a toda la comunidad.

En el modelo de educación intercultural socio-crítico-reconstructivista, el profesor debe ser competente para preparar a los alumnos para vivir en una sociedad donde la diversidad cultural se hace presente. La lengua materna es adquisición y punto de apoyo importante en todo el aprendizaje; se hace necesario desarrollar en los alumnos el gusto y la capacidad de trabajar en la construcción conjunta de una sociedad donde las diferencias culturales se consideren una riqueza común y no factor de división; reconocer y aceptar el pluralismo cultural como una realidad social y contribuir a la instauración de una sociedad de igualdad de derechos y equidad, contribuyendo al establecimiento de relaciones interétnicas armoniosas.

Sedano (1998: 85), presenta la formación y fortalecimiento en la escuela y en la sociedad de los valores humanos de la igualdad, respeto, tolerancia, pluralismo, cooperación y corresponsabilidad social como principios básicos de la educación intercultural. Añade también, el reconocimiento del derecho personal de cada alumno a recibir la mejor educación diferenciada, un cuidado especial a la formación de su identidad personal, el reconocimiento positivo de las diversas culturas y lenguas y de su necesaria presencia y cultivo en la escuela, la atención a la 
diversidad y respeto a las diferencias, sin etiquetar ni definir a nadie en virtud de éstas, la no segregación en grupos aparte, la lucha activa contra toda manifestación de racismo o discriminación, el intento de superación de estereotipos y prejuicios, la mejoría del éxito escolar de los alumnos de minorías étnicas, una gestión democrática y participación activa de los alumnos y los padres de los alumnos en la escuela, el incremento de relaciones positivas entre los diversos grupos étnicos, y por último, la inserción activa de la escuela en la comunidad local.

Para él la educación intercultural implica la formación sistemática de todo educando en la comprensión de la diversidad cultural de la sociedad actual, el aumento de la capacidad de comunicación entre personas de diversas culturas, la creación de actitudes favorables a la diversidad de culturas y el incremento de interacción social entre personas y grupos culturalmente distintos.

El modelo planteado sólo podrá realizarse mejor si los profesores asumen la concepción del profesional reflexivo, que investiga, analiza y reflexiona sobre su práctica. (Schön, 2000; Stenhouse, 1996; Novoa, 1995 y Zeichner, 1998). Ya que no es suficiente que el profesorado cuente con un repertorio de conocimientos culturales y pedagógicos, se hace necesario un cambio de actitudes, creencias, ideas y preconcepciones, que en definitiva, son las que influyen en el modo de realizar el área educativa.

Un profesor que desea adherirse al movimiento para lograr una educación intercultural debe cultivar las actitudes de respeto a la persona humana, respeto a todo el pueblo y su propia cultura, respeto a la autonomía de los alumnos, tolerancia de ideas y conductas individuales y grupales que no sean contrarias a los derechos humanos, sentido crítico frente a las leyes y situaciones injustas que afectan los derechos humanos, lucha activa contra la discriminación racial, aceptación interpersonal, superación del egoísmo y etnocentrismo, cooperación activa en la construcción social, corresponsabilidad social y aceptación positiva de las diferencias, entre otras.

En cuanto a los conocimientos nos gustaría acentuar que el profesor debe ser capaz de elaborar diseños curriculares y unidades didácticas de inspiración intercultural, hacer uso de diferentes metodologías y técnicas de enseñanza-aprendizaje que fomenten relaciones interculturales constructivas: aprendizaje por proyecto de trabajo, aprendizaje cooperativo; diseñar y utilizar estrategias que favorezcan las relaciones interculturales en el aula, a partir de cambios organizativos y funcionales; desarrollar destrezas de comunicación con las familias y otros profesionales; tales como desarrollar habilidades para detectar prejuicios y estereotipos contenidos en los materiales de enseñanza y en los medios de comunicación social; diagnosticar valores, necesidades educativas y estilos de aprendizaje de los alumnos; dominar estrategias para una pedagogía del éxito escolar, que incremente el rendimiento de todos los alumnos, especialmente de los pertenecientes a minorías marginadas (Nieto, 2002: 90).

La formación del profesorado es un reto para la educación intercultural dentro de una perspectiva socio-crítica y socio-reconstructivista, en una sociedad cada vez más desafiada por los cambios sociales. 


\section{NOTAS}

${ }^{1}$ Según Sabariego (2002, p. 74) existe un consenso general de los autores Aguado (1991), Camilleri (1992), Carbonell (1977), Davies (1996), Etxeberria (1992), Flecha (1994), Galino y Escribano (1990), Johansson (1996), Molina (1994), Muñoz Sedano (1997), Puig Moreno (1991), Ruy (1996), Tuvilla (1998) a reconocer que el término intercultural tiene significado diferente de multicultural. Esto expresa una situación de hecho, la situación real de una sociedad con varios grupos culturales que mantienen una suficiente cohesión entre ellos de acuerdo con un cierto número de valores y normas, mientras que interculturalidad afirma expresamente la realidad del diálogo, la reciprocidad, la interdependencia, o un deseo, un método de intervención, por medio del cual la interacción entre culturas diferentes sea una fuente de enriquecimiento recíproco.

El término educación multicultural puede seguir la idea de una sociedad construida como mosaico, dividida, estática, no dialógica, no interactiva, impulsando una coexistencia forzosa, de rechazo o de ignorancia mutua, capaz de conducir la formación de guetos. En este estudio utilizaremos el término educación intercultural o interculturalismo para designar el carácter de un proceso educativo dinámico, de interacción, de intercambio, de apertura y solidaridad efectiva, desde el reconocimiento de los valores, de los modos de vivir, las representaciones simbólicas entre culturas homogéneas o no.

${ }^{2}$ Empleamos el término educación multicultural aquí, porque nos referimos a los modelos (cerrados o abiertos, dinámicos y no dinámicos, interactivo y no interactivo) de educación en contexto multicultural.

\section{BIBLIOGRAFÍA}

ABDALLAH - PRETEILLE, M., (2001): La educación intercultural, Idea Books, Barcelona.

BARTOLOMÉ, M., (1995): "La escuela multicultural: del diagnóstico a una propuesta de cambio", Revista de Educación (307), 75 - 125.

CASTAÑO, F. J. G. y MARTÍNEZ, A. G., (1999): Lecturas para educción intercultural, Trotta, Madrid.

GARCÍA y SÁEZ (1998): Del racismo a la interculturalidad. Competencia de la Educación, Narcea, Madrid.

GERALDI, C., (1998): Refletindo com Zeichner: um encontro orientado por preocupações políticas e teóricas epistemológicas, Mercado das Letras, Campinas, São Paulo.

JORDAN, J., (1998): La escuela multicultural - un reto para el profesorado, Paidós, Barcelona.

MCLAREN, P., (2000): Multiculturalismo revolucionário - pedagogia do dissenso para o novo milênio, Artmed, Porto Alegre. 
MOREIRA, A F. B., "Currículo, diferença cultural y diálogo", Revista Educação e Sociedade, Dossiê "diferentes", n. 79, ano XXIII, agosto, 2002. pp. 15-38. MUÑOZ, R. M. et al. (2002): La mejora de la escuela: un cambio de mirada, Octaedro, Barcelona.

NIETO, A. C. del C. et al. (2002): La educación intercultural: un reto en el presente de Europa, Consejeria de Educación, Comunidad de Madrid.

NOVOA, A., (1996): "As Ciências da Educação e os processos de mudança", Pedagogia, ciência da educação?, São Paulo, Cortez.

— (1998): Os professores e a sua formação, Publicações D.Quixote, LTDA, Lisboa.

(1995): "Para uma análise das instituições escolares", As organizações escolares em análise - $2^{\mathrm{a}}$ edição.

PÉREZ de CUELLAR, J., (1997): La nostra diversitat creativa. Informe de la Comissió Mundial sobre Cultura y Desarrollo, Centre UNESCO de Catalunya, Orial Mediterrânia, Barcelona.

RODRÍGUEZ, J. M., (1995): Formación de profesores y prácticas de enseñanza, Universidad de Huelva, Huelva.

SABARIEGO, M., (2002): La educación intercultural, ante los retos del siglo XXI, Descleé, Bilbao.

SCHÖN, D. A., (1992): La formación de profesionales reflexivos. Hacia un nuevo diseño de la enseñanza y el aprendizaje en las profesiones, Paidós, Barcelona.

STENHOUSE, L. (1996): La investigación como base de la enseñanza, Ediciones Morata, Madrid.

ZEICHNER, K. (1998): "Para além da divisão entre professor pesquisador e pesquisador acadêmico", Geraldi, C., Cartografias do Professor, Mercado das Letras, Campinas, Sao Paulo, pp. $207-235$. 